\title{
Efeito da COVID-19 na artroplastia de ombro em um centro médico terciário na cidade de Nova York
}

\section{Effect of COVID-19 on Shoulder Arthroplasty at a Tertiary Care Medical Center in New York City}

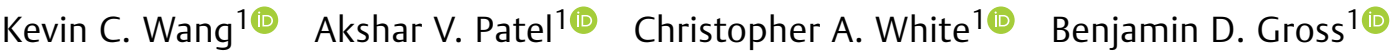 \\ Bradford O. Parsons ${ }^{10}$ Paul J. Cagle ${ }^{1(1)}$ \\ ${ }^{1}$ Departamento de Cirurgia Ortopédica, Icahn School of Medicine at \\ Mount Sinai, New York City, New York, Estados Unidos \\ Endereço para correspondência Paul J. Cagle, MD, 425 West 59th \\ Street, New York, NY 10019, United States \\ (e-mail: Paul.Cagle@mountsinai.org).
}

Rev Bras Ortop 2023;58(1):121-126.

\section{Resumo}

Palavras-chave

- artroplastia do ombro

- COVID-19

- infecções por coronavírus

- procedimentos cirúrgicos eletivos

- período perioperatório

- período pósoperatório
Objetivo A pandemia de COVID-19 causou uma pausa sem precedentes em cirurgias eletivas, inclusive artroplastia de ombro. Procuramos determinar as possíveis diferenças clínicas e/ou demográficas entre os pacientes que realizaram artroplastia de ombro durante a pandemia em comparação com o ano anterior (2019).

Métodos Os registros institucionais foram consultados para obtenção de informações sobre pacientes submetidos a artroplastia de ombro entre $1^{\circ}$ de março a $1^{\circ}$ de julho de 2019 e 2020. Dados demográficos, amplitude de movimento, duração da cirurgia, tempo de hospitalização, condições à alta e manejo pós-operatório foram analisados.

Resultados O tempo médio de cirurgia foi de $160 \pm 50$ minutos em 2020 e de $179 \pm 54$ minutos em $2019(p=0,13)$. O tempo médio de internação foi de $36 \pm 13$ horas em 2020 e de $51 \pm 40$ horas em 2019 ( $p=0,04)$. Em 2019, 96\% dos pacientes fizeram fisioterapia, enquanto $71 \%$ o fizeram em $2020(p=0,003)$. Todos os pacientes de 2019 e $86 \%$ dos pacientes de 2020 participaram do acompanhamento pósoperatório presencial $(p=0,006)$. Os pacientes de 2019 retornaram para a consulta médica em média $14 \pm 11$ dias após a cirurgia; os pacientes de 2020 retornaram para o acompanhamento em $25 \pm 25$ dias $(p=0,10)$. A amplitude de movimento, a idade, a pontuação da American Society of Anesthesiologists (ASA, na sigla em inglês) e as taxas de complicações não diferiram entre as coortes.

Conclusão Os pacientes submetidos a cirurgia na fase inicial da pandemia eram demográfica e clinicamente semelhantes aos pacientes de 2019. No entanto, o tempo de internação diminuiu de forma significativa durante a pandemia de COVID-19. O acompanhamento pós-operatório e a fisioterapia foram adiados em 2020, mas isso não

Trabalho desenvolvido no Departamento de Cirurgia Ortopédica, Icahn School of Medicine at Mount Sinai, New York City, NY.

recebido

19 de Maio de 2021

aceito após revisão

13 de Agosto de 2021

article Publicado on-line

Outubro 25, 2021
DOI https://doi.org/

10.1055/s-0041-1735950.

ISSN 0102-3616.
(C) 2021. Sociedade Brasileira de Ortopedia e Traumatologia. All rights reserved.

This is an open access article published by Thieme under the terms of the Creative Commons Attribution-NonDerivative-NonCommercial-License, permitting copying and reproduction so long as the original work is given appropriate credit. Contents may not be used for commercial purposes, or adapted, remixed, transformed or built upon. (https://creativecommons.org/ licenses/by-nc-nd/4.0/)

Thieme Revinter Publicações Ltda., Rua do Matoso 170, Rio de Janeiro, RJ, CEP 20270-135, Brazil 


\section{Abstract}

\section{Keywords}

- arthroplasty, replacement, shoulder

- COVID-19

- coronavirus infections

- elective surgical procedures

- perioperative period

- postoperative period levou a diferenças nas taxas de complicações ou de reinternações em comparação às da coorte de 2019.

Nível de Evidência III.

Objective The COVID-19 pandemic led to an unprecedented pause in elective surgeries, including shoulder arthroplasty. We sought to determine whether clinical and/or demographic differences would be seen between patients who presented for shoulder arthroplasty during the pandemic compared with the previous year (2019). Methods Institutional records were queried for patients who underwent shoulder replacement between March 1 and July 1 of 2019 and 2020. Demographics, range of motion, surgical duration, hospitalization time, discharge disposition, and postoperative management were analyzed.

Results The mean duration of surgery was $160 \pm 50$ minutes in 2020 and $179 \pm 54$ minutes in $2019(p=0.13)$. The mean hospitalization time was $36 \pm 13$ hours in 2020 and $51 \pm 40$ hours in $2019(p=0.04)$. In 2019, 96\% of the patients participated in physical therapy, while $71 \%$ did it in $2020(p=0.003)$. A total of $100 \%$ of the 2019 patients and $86 \%$ of the 2020 patients participated in an in-person postoperative follow-up $(p=0.006)$. The 2019 patients reported for an office visit on average $14 \pm 11$ days after surgery; the 2020 patients waited $25 \pm 25$ days to return for a follow-up $(p=0.10)$. Range of motion, age, American Society of Anesthesiologists (ASA) scores, and complication rates did not differ between the cohorts.

Conclusion Patients presenting for surgery during the initial phase of the pandemic were demographically and clinically similar to 2019 patients. However, the length of stay was significantly reduced during the COVID-19 pandemic. Postoperative follow-up and physical therapy were delayed in 2020 , but this did not lead to differences in complication or readmission rates compared with those of the 2019 cohort.

Level of Evidence III.

\section{Introdução}

O primeiro caso de COVID-19 foi relatado em Nova York em $1^{\circ}$ de março de 2020 , e a região rapidamente se tornou o epicentro global da doença. Em 2 semanas, o Surgeon General dos Estados Unidos aconselhou os hospitais a interromper temporiamente as cirurgias eletivas para minimizar a propagação do vírus e maximizar os suprimentos de saúde existentes. Isto levou ao advento de novos protocolos de tratamento e diretrizes de distanciamento social que reduziram significativamente a hospitalização, a disseminação e a mortalidade da doença.

O impacto econômico da interrupção de cirurgias ortopédicas eletivas foi significativo. Os procedimentos eletivos, inclusive as principais cirurgias ortopédicas, como as artroplastias, são historicamente responsáveis por boa parte da renda dos sistemas de saúde., ${ }^{1,2}$ Enquanto o mundo se recupera do impacto generalizado da pandemia de COVID19 , a cirurgia eletiva desempenha um papel fundamental na restauração da estabilidade econômica dos sistemas de saúde. No entanto, mudanças importantes devem ocorrer para assegurar a segurança de pacientes e profissionais e a boa administração dos recursos de saúde. A American Academy of Orthopaedic Surgeons (AAOS, na sigla em inglês) identificou quatro níveis de procedimentos ortopédicos durante a pandemia - A (emergenciais), B (urgentes), C (urgentes/eletivos) e D (eletivos). ${ }^{3}$ Cada nível pode ser retomado com as precauções adequadas aos diferentes estágios de recuperação da pandemia.

Os algoritmos atuais recomendam a consideração cuidadosa da urgência do procedimento, dos fatores de risco do paciente (ou seja, idade, comorbidades médicas e outros fatores) e dos recursos necessários (ou seja, necessidades de internação ou alta) com a aplicação adequada da triagem pré-operatória e teste de COVID. ${ }^{3,4}$ Além disso, a vontade do paciente em se submeter à cirurgia é um fator essencial para a retomada dos procedimentos eletivos. Em um estudo recentemente publicado, $27 \%$ dos pacientes que responderam a um questionário online durante o início da pandemia, em maio de 2020, afirmaram que estariam dispostos a se submeter a procedimentos eletivos no primeiro mês de reabertura; no entanto, $61 \%$ dos entrevistados relatarem medo de se expor ou contrair o vírus. ${ }^{5}$ Isto sugere que, apesar dos temores em relação à COVID-19, a demanda por cirurgias eletivas começa a se recuperar.

A artroplastia total de ombro (ATO) é um procedimento eletivo que, embora menos realizado do que a artroplastia de joelho e quadril, cresce em prevalência. Este procedimento pode dar alívio significativo da dor da artrite debilitante do ombro, em especial nos primeiros 6 meses após a cirurgia. ${ }^{6}$ 
Antes da pandemia, o tempo médio de internação para ATO já caía de forma contínua (com uma redução $>50 \%$ entre 1993 e 2007) e houve maior interesse na realização do procedimento em ambulatório em uma tentativa de diminuição de custos para o paciente e o sistema de saúde. ${ }^{7}$ Assim, a incidência de ATO ambulatorial tem aumentado constantemente, quase dobrando entre 2010 e 2014, sem nenhuma evidência de aumento das taxas de reinternação ou de complicações em pacientes escolhidos de forma adequada. Esta transição para procedimentos ambulatoriais gerou uma economia de quase 4.000 dólares por cirurgia. ${ }^{8}$ No entanto, parte significativa destes procedimentos continua sendo realizada em regime de internação, o que pode dificultar o retorno à capacidade anterior. Uma pesquisa anterior observou um aumento do tempo de internação e probabilidade de alta para unidade de saúde em pacientes $>65$ anos, do sexo feminino, com obesidade ou diabetes e submetidos à artroplastia total reversa do ombro (ATOr). ${ }^{9}$ Algumas destas características demográficas, especificamente idade avançada e comorbidades médicas, são observadas em pacientes com maior risco de desenvolvimento de COVID-19 grave.

O objetivo do presente estudo é investigar a diferença demográfica entre pacientes submetidos à ATO em 2020, durante a pandemia de COVID-19, em comparação com pacientes submetidos ao mesmo procedimento em 2019, um ano antes da pandemia. Devido à tendência atual de realização de ATO em regime ambulatorial, as atitudes do paciente em relação à cirurgia e a ênfase na alocação eficiente de recursos e redução de risco para os pacientes durante a pandemia de COVID-19, acreditamos que os indivíduos submetidos à artroplastia de ombro durante a pandemia serão mais jovens, mais saudáveis, ficarão internados por menos tempo e apresentarão maior probabilidade de receber alta domiciliar do que para um centro de cuidados em curto prazo em comparação com o ano anterior à pandemia.

\section{Materiais e Métodos}

\section{Delineamento Experimental e Amostra}

O presente estudo foi aprovado por nosso conselho de revisão institucional. Este é um estudo retrospectivo de pacientes submetidos à ATO ou ATOr no início da pandemia de COVID19 , definido como o período de $1^{\circ}$ de março a $1^{\circ}$ de julho de 2020. O primeiro caso de COVID-19 foi confirmado na cidade de Nova York em $1^{\circ}$ de março de 2020 , e $1^{\circ}$ de julho de 2020 foi o primeiro dia em que $<1.000$ novos casos foram relatados. A realização de cirurgias eletivas foi interrompida em 14 de março de 2020 de acordo com a recomendação do Surgeon General dos Estados Unidos e retomada em 8 de junho de 2020. Uma coorte de pacientes que se submeteram a ATO ou ATOr entre $1^{\circ}$ de março e $1^{\circ}$ de julho de 2019 foi usada como grupo comparativo. Os registros institucionais foram consultados usando os códigos de Current Procedural Terminology (CPT, na sigla em inglês) 23470, 23472, 23473 e 23474.

\section{Análise Estatística}

Todas as análises de dados e testes estatísticos foram realizados no software R, versão 4.0.5 (R Foundation, Viena,
Áustria). As variáveis categóricas foram apresentadas como frequências $(n / N)$ e porcentagens, enquanto as variáveis contínuas foram apresentadas como média ( \pm desvio padrão [DP]). A normalidade foi determinada pelo teste de ShapiroWilk. As análises univariadas foram realizadas com o teste de X2 no caso de variáveis categóricas e com o teste t de Student ou o teste U de Mann-Whitney (soma dos postos de Wilcoxon) para variáveis contínuas. Todos os valores de $p<0,05$ foram considerados estatisticamente significativos.

\section{Resultados}

\section{População do Estudo}

O estudo identificou 21 pacientes em 2020 e 50 pacientes em 2019 que foram submetidos à ATO. Destes pacientes, 50 e $62 \%$ eram do sexo masculino nas coortes de 2019 e 2020, respectivamente $(p=0,36)$. A média de idade em 2019 era de $69 \pm 9$ anos, e de $68 \pm 7$ anos em $2020(p=0,71)$. Em 2020, 10 ATOs e 11 ATOrs foram realizadas, contra 16 ATOs, 30 ATOrs, 1 hemiartroplastia e 3 cirurgias de revisão em $2019(p=0,16)$ (-Tabela 1).

Não houve diferença significativa nos dados demográficos dos pacientes entre as coortes de 2019 e 2020 . A elevação anterior média pré-operatória era de $101^{\circ} \pm 41^{\circ}$ em 2019 e de $115^{\circ} \pm 36^{\circ}$ em $2020(p=0,19)$. A rotação externa média pré-operatória era de $26^{\circ} \pm 19^{\circ}$ em 2019 e de $26^{\circ} \pm 15^{\circ}$ em $2020(p=0,92)$. A pontuação média da American Society of Anesthesiologists (ASA, na sigla em inglês) dos pacientes de 2019 era $2,4 \pm 0,70$, e $2,5 \pm 0,59$ em 2020 ( $p=0,74)$. No total, $58 \%$ dos pacientes de 2019 e $52 \%$ dos pacientes de 2020 apresentaram pontuação ASA $\leq 2$. Em 2020, 24\% da coorte usava o Medicaid como seguro principal, enquanto apenas $8 \%$ da coorte de 2019 o usava $(p=0,12)$. Em 2020, havia 3 casos urgentes e 18 eletivos; em 2019, foram 43 casos eletivos e 7 casos urgentes $(p=0,98)$. Um total de $28 \%$ dos pacientes em 2019 e 33\% dos pacientes de 2020 já haviam sido submetidos a uma cirurgia no ombro acometido $(p=0,65)$ (-Tabela 1 ).

\section{Variáveis de Desfechos}

Houve uma diferença na duração da cirurgia, mas sem significância estatística. Em 2019, o tempo médio de cirurgia foi de $179 \pm 54$ minutos e, em 2020 , de $160 \pm 50$ minutos $(p=0,13)$. A diferença no tempo de internação entre os dois anos foi estatisticamente significativa. Em 2019, os pacientes ficam em média $51 \pm 40$ horas no hospital; contudo, em 2020 , a estadia média foi de $36 \pm 13$ horas $(p=0,04)$. Em $2019,12 \%$ dos pacientes receberam alta para uma unidade de tratamento subagudo e $88 \%$ tiveram alta domiciliar; em 2020, 9,5\% dos pacientes receberam alta para uma unidade de tratamento subagudo e 90,5\% receberam alta domiciliar $(p=0,76)$ (-Tabela 2 ).

O tratamento pós-operatório após ATO ou ATOr também variou de forma significativa entre os 2 anos. Em 2019, 96\% dos pacientes participaram de fisioterapia presencial, enquanto 71\% o fizeram em 2020 ( $p=0,003)$. Em 2019, 100\% dos pacientes $(n=50)$ compareceram a uma consulta pós-operatória com seu cirurgião, enquanto $86 \%$ dos pacientes $(n=21)$ fizeram o mesmo em 2020 ( $p=0,006)$. Dentre os 3 pacientes 
124 Efeito da COVID-19 na artroplastia de ombro Wang et al.

Tabela 1 Dados demográficos dos pacientes ( $n=50$ em 2019; $n=21$ em 2020)

\begin{tabular}{|c|c|c|c|}
\hline Característica & 2019 (DP) & 2020 (DP) & valor-p \\
\hline $\begin{array}{l}\text { Idade à cirurgia } \\
\text { (anos) }\end{array}$ & $69,3 \pm 8,7$ & $68,3 \pm 7,4$ & 0,71 \\
\hline \multicolumn{4}{|l|}{ Gênero } \\
\hline Masculino & $25(50,0 \%)$ & $13(61,9 \%)$ & \multirow[t]{2}{*}{0,36} \\
\hline Feminino & $25(50,0 \%)$ & $8(38,1 \%)$ & \\
\hline \multicolumn{4}{|l|}{ Pontuação ASA } \\
\hline 1 & $3(6,0 \%)$ & $1(4,8 \%)$ & \multirow[t]{4}{*}{0,74} \\
\hline 2 & $26(52,0 \%)$ & $10(47,6 \%)$ & \\
\hline 3 & $19(38,0 \%)$ & $10(47,6 \%)$ & \\
\hline 4 & $2(4,0 \%)$ & $0(0,0 \%)$ & \\
\hline \multicolumn{4}{|l|}{ Plano de saúde } \\
\hline Medicaid & $4(8,0 \%)$ & $5(23,8 \%)$ & \multirow[t]{4}{*}{0,12} \\
\hline Medicare & $25(50,0 \%)$ & $10(47,6 \%)$ & \\
\hline Medicare/Privado & $7(14,0 \%)$ & $0(0,0 \%)$ & \\
\hline Privado & $14(28,0 \%)$ & $6(28,6 \%)$ & \\
\hline \multicolumn{4}{|l|}{ Diabetes } \\
\hline Sim & $10(20,0 \%)$ & $3(14,3 \%)$ & \multirow[t]{2}{*}{0,57} \\
\hline Não & $40(80,0 \%)$ & $18(85,7 \%)$ & \\
\hline \multicolumn{4}{|l|}{ Hipertensão } \\
\hline Sim & $32(64,0 \%)$ & $14(66,7 \%)$ & \multirow[t]{2}{*}{0,83} \\
\hline Não & $18(36,0 \%)$ & $7(33,3 \%)$ & \\
\hline \multicolumn{4}{|c|}{ Cirurgia prévia no ombro } \\
\hline Sim & $14(28,0 \%)$ & $7(33,3 \%)$ & \multirow[t]{2}{*}{0,65} \\
\hline Não & $36(72,0 \%)$ & $14(66,7 \%)$ & \\
\hline \multicolumn{4}{|l|}{ Tipo de ATO } \\
\hline Anatômica & $30(60,0 \%)$ & $10(47,6 \%)$ & \multirow[t]{3}{*}{0,16} \\
\hline Reversa & $16(32,0 \%)$ & $11(52,4 \%)$ & \\
\hline Hemiartroplastia & $4(8,0 \%)$ & $0(0,0 \%)$ & \\
\hline \multicolumn{4}{|c|}{ Eletiva ou Emergencial } \\
\hline Eletiva & $43(86,0 \%)$ & $18(85,7 \%)$ & \multirow[t]{2}{*}{0,98} \\
\hline $\begin{array}{l}\text { Emergência } \\
\text { médica }\end{array}$ & $7(14,0 \%)$ & $3(14,3 \%)$ & \\
\hline $\begin{array}{l}\text { Elevação anterior } \\
\text { pré-operatória }\end{array}$ & $100,7^{\circ}(41,3)$ & $114,5^{\circ}(35,6)$ & 0,19 \\
\hline $\begin{array}{l}\text { Rotação externa } \\
\text { pré-operatória }\end{array}$ & $25,8^{\circ}(19,1)$ & $26,3^{\circ}(15,2)$ & 0,74 \\
\hline
\end{tabular}

Abreviações: ASA, American Society of Anesthesiologists; ATO, artroplastia de ombro; DP, desvio padrão.

que não retornaram para a consulta em 2020, 1 foi atendido pelo cirurgião por telefone $(n=1)$, outro pelo serviço de telemedicina $(n=1)$, e o terceiro não compareceu a nenhum acompanhamento. Em 2019, os pacientes compareceram ao consultório em média $14 \pm 11$ dias após a data da cirurgia; em 2020, os pacientes retornaram para consulta $25 \pm 25$ dias após a cirurgia $(p=0,10)$. Os pacientes de 2020 também utilizaram mais os serviços de telemedicina (-Tabela $\mathbf{3}$ ).
Tabela 2 Características perioperatórias do paciente $(n=50$ em 2019; $n=21$ em 2020)

\begin{tabular}{|c|c|c|c|}
\hline Característica & 2019 (DP) & 2020 (DP) & valor-p \\
\hline $\begin{array}{l}\text { Duração da cirurgia } \\
\text { (minutos) }\end{array}$ & $178,7(54,3)$ & $159,5(50,1)$ & 0,13 \\
\hline $\begin{array}{l}\text { Tempo de internação } \\
\text { (horas) }\end{array}$ & $51,3(40,0)$ & $36,3(12,6)$ & 0,04 \\
\hline \multicolumn{4}{|l|}{ Transfusão de sangue } \\
\hline Sim & $1(2,0 \%)$ & $0(0,0 \%)$ & \multirow[t]{2}{*}{0,51} \\
\hline Não & $49(98,0 \%)$ & $21(100 \%)$ & \\
\hline \multicolumn{4}{|l|}{ Complicações cirúrgicas } \\
\hline Sim & $4(8,0 \%)$ & $2(9,5 \%)$ & \multirow[t]{2}{*}{0,83} \\
\hline Não & $46(92,0 \%)$ & $19(90,5 \%)$ & \\
\hline
\end{tabular}

Abreviação: DP, desvio padrão.

\section{Discussão}

A pandemia de COVID-19 causou uma interrupção sem precedentes na realização de cirurgias eletivas em março de 2020. Com sede em Nova York, nossa instituição foi significativamente afetada como epicentro anterior da pandemia global. As cirurgias eletivas foram interrompidas no início de março e, a princípio, apenas os casos emergenciais foram operados. Após este período de interrupção, os procedimentos eletivos foram retomados de forma lenta, em fases, com testes específicos de COVID-19 e protocolos de

Tabela 3 Características pós-operatórias do paciente $(n=50$ em 2019; $n=21$ em 2020)

\begin{tabular}{|c|c|c|c|}
\hline Característica & 2019 (DP) & 2020 (DP) & valor-p \\
\hline \multicolumn{4}{|l|}{ Destino pós-operatório } \\
\hline Domicílio & $44(88,0 \%)$ & $19(90,5 \%)$ & \multirow[t]{2}{*}{0,76} \\
\hline Tratamento subagudo & $6(12,0 \%)$ & $2(9,5 \%)$ & \\
\hline \multicolumn{4}{|l|}{ Cirurgia de revisão } \\
\hline Sim & $3(6,0 \%)$ & $2(9,5 \%)$ & \multirow[t]{2}{*}{0,60} \\
\hline Não & $47(94,0 \%)$ & $19(90,5 \%)$ & \\
\hline \multicolumn{4}{|l|}{ Reinternação } \\
\hline Sim & $6(12,0 \%)$ & $2(9,5 \%)$ & \multirow[t]{2}{*}{0,76} \\
\hline Não & $44(88,0 \%)$ & $19(90,5 \%)$ & \\
\hline \multicolumn{4}{|c|}{ Método de consulta pós-operatória } \\
\hline $\begin{array}{l}\text { Consulta de } \\
\text { telemedicina }\end{array}$ & $0(0,0 \%)$ & $3(14,3 \%)$ & \multirow[t]{2}{*}{0,006} \\
\hline Consulta presencial & $50(100 \%)$ & $18(85,7 \%)$ & \\
\hline $\begin{array}{l}\text { Tempo entre a } \\
\text { cirurgia e a consulta } \\
\text { pós-operatória (dias) }\end{array}$ & $14,1(11,4)$ & $25,4(24,7)$ & 0,10 \\
\hline \multicolumn{4}{|l|}{ Fisioterapia } \\
\hline Sim & $48(96,0 \%)$ & $15(71,4 \%)$ & \multirow[t]{2}{*}{0,003} \\
\hline Não & $2(4,0 \%)$ & $6(28,6 \%)$ & \\
\hline
\end{tabular}

Abreviação: DP, desvio padrão. 
controle de infecção. O presente estudo busca compreender as possíveis diferenças significativas nos dados demográficos ou nas características pós-operatórias iniciais dos pacientes submetidos à ATO durante a primeira fase da pandemia em relação ao ano anterior. Nossa hipótese é que os pacientes de coortes de baixo risco de COVID-19 e com doença mais avançada no ombro seriam submetidos à cirurgia durante a pandemia.

A percepção do público sobre a retomada das cirurgias eletivas foi um fator significativo na determinação de quais pacientes seriam submetidos a estes procedimentos durante a pandemia. Em um estudo recente, Moverman et al..$^{5}$ observaram que homens e falantes de inglês se sentiam significativamente mais confortáveis ao optarem pela cirurgia eletiva após o fim das restrições. No entanto, na nossa pesquisa, não houve diferença significativa no número de homens e mulheres que optaram pela realização de ATO entre 2019 e 2020 (50\% em 2019 e $62 \%$ em 2020; $p=0,36$ ). Não houve diferença significativa no número de falantes de inglês em qualquer coorte (98\% em 2019 e 92\% em 2020; $p=0,97$ ).

Nossa hipótese inicial era que os pacientes que optassem pela artroplastia eletiva do ombro em 2020 apresentariam pontuações ASA menores e seriam mais jovens. Outra hipótese era que os pacientes que optassem pela artroplastia do ombro em 2020 apresentariam pior amplitude de movimento pré-operatória. Essas hipóteses refletiram nossas suposições de que os pacientes em grupos de menor risco de desenvolvimento de complicações da COVID-19 e piores doenças ou limitações do ombro optariam pela cirurgia durante a pandemia. No entanto, estas variáveis pré-operatórias não foram significativamente diferentes entre as duas coortes. Na verdade, não encontramos diferenças significativas em nenhuma das variáveis demográficas pré-operatórias entre as coortes de 2019 e 2020 . Além disso, houve uma redução significativa de volume entre as coortes de 2019 e 2020 (52\% menos procedimentos de artroplastia de ombro), o que é atribuível à pausa de 3 meses em cirurgias eletivas. Isso é semelhante às reduções de volume de casos ortopédicos eletivos relatadas por outras instituições. ${ }^{10}$ Também observamos um declínio nos procedimentos de artroplastia relacionados a traumatismos (5 em 2019 e 1 em 2020). Isto refletiu um declínio nos casos de traumatismo ortopédico durante a interrupção. ${ }^{10,11}$

É importante ressaltar que a nossa pesquisa observou uma tendência de significado estatístico na duração da cirurgia $(178,7 \pm 54,3$ em 2019 e 159,5 $\pm 50,1$ em 2020; $p=0,13)$. Embora parte desta diferença possa ser devida ao menor número de procedimentos de revisão realizados durante a pandemia, o menor volume cirúrgico e a maior eficiência da alocação de recursos também podem ter contribuído. Observamos uma diminuição significativa no tempo de permanência pós-operatória entre as 2 coortes (51,3 \pm 40 ,0 horas em 2019 e $36,3 \pm 12,6$ horas em 2020; $p=0,04)$. Em 2020, nenhum paciente permaneceu no hospital $>72$ horas. Em 2019, 12 pacientes permaneceram $>72$ horas (72 a 100 horas, $n=7$; 100 a 200 horas, $n=3 ; 200$ a 300 horas, $n=1$; e $>300$ horas, $n=1)$. O destino pós-operatório dos pacientes não foi significativamente diferente, com 88 e $91 \%$ indo para casa após a cirurgia em 2019 e 2020, respectivamente $(p=0,76)$. Durante a pandemia de COVID-19, uma combinação de recursos hospitalares tributados e o desejo do paciente de logo deixar o hospital pode ter contribuído para o menor tempo até a alta, alinhando seus incentivos. Pesquisas anteriores observaram uma melhora no manejo pré-operatório que levou à redução do tempo de internação durante a pandemia de COVID-19, mas o presente estudo é o primeiro de que temos conhecimento a demonstrar a diminuição do tempo de internação pós-operatória na literatura ortopédica. ${ }^{12}$

Uma revisão sistemática anterior observou que o tempo de internação e o tipo de alta após a ATO foram influenciados por uma série de fatores: sexo feminino, obesidade, idade $>65$ anos e ATOr foram associados à internação mais longa ou à necessidade de alta para outra instituição. ${ }^{9}$ No nosso estudo, o tempo de internação melhorou de 2,1 $\pm 1,5$ dias em 2019 para 1,5 \pm 0,5 dias em 2020. Estes intervalos de 2019 são comparáveis aos citados na literatura. ${ }^{13,14}$ Notavelmente, nenhum aumento nas taxas de readmissão ou de complicações em 30 ou 90 dias foi observado em nossas coortes.

No período pós-operatório, um número significativamente menor de pacientes participou de fisioterapia formal em 2020 (71\%) em comparação com 2019 (96\%) $(p=0,003)$. Além disso, menos pacientes retornaram para a consulta pósoperatória presencial e o tempo entre a cirurgia até a primeira consulta pós-operatória foi ligeiramente maior durante a pandemia. A literatura sugere que a fisioterapia pós-operatória imediata proporciona redução da dor e aumento da função em curto prazo, mas estes benefícios se dissipam em um acompanhamento de longo prazo. ${ }^{15}$ Não houve aumento da taxa de complicações precoces, apesar da diferença no acompanhamento pós-operatório imediato; no entanto, nossa pesquisa é insuficiente para tirar quaisquer conclusões robustas sobre as complicações pós-operatórias. Juntos, estes resultados sugerem que, apesar da semelhança dos dados demográficos de pacientes de 2019 e 2020, os indivíduos submetidos a ATO puderam ter alta hospitalar de forma consistente no início do período pós-operatório durante a pandemia. Isto pode ser devido a diversos fatores, inclusive a motivação do paciente, $o$ alinhamento dos incentivos para a alta entre pacientes e profissionais e o aumento de recursos hospitalares disponíveis, como assistentes sociais, para auxiliar o processo de alta.

A presente pesquisa tem várias limitações, como o pequeno tamanho da amostra e a natureza retrospectiva do estudo. Além disso, todos os pacientes da nossa coorte eram de uma única região urbana que foi duramente atingida pela pandemia de COVID-19 e os resultados podem não ser passíveis de generalização para outras populações. Além disso, não havia desfechos relatados pelo paciente.

\section{Conclusão}

A pandemia de COVID-19 provocou uma pausa sem precedentes na realização de cirurgias eletivas a partir de março de 2020. Apesar da percepção pública sobre a segurança das cirurgias eletivas durante a pandemia, não notamos diferenças nas características clínicas ou demográficas dos 
pacientes que se submeteram a ATO no início da pandemia em comparação com o ano anterior. No entanto, observamos reduções no tempo de cirurgia, embora sem significado estatístico, e no tempo de internação, sugerindo um movimento coordenado em direção à redução da hospitalização durante a pandemia. Além disso, apesar da maior espera para o retorno ao acompanhamento pós-operatório e menor participação na fisioterapia em 2020, os pacientes não apresentaram diferenças nas taxas de readmissão, de revisão ou de complicações em seus respectivos anos.

\section{Suporte Financeiro}

A presente pesquisa não recebeu nenhuma bolsa específica de agências de fomento nos setores públicos, comerciais ou sem fins lucrativos.

\section{Conflitos de Interesses \\ Paul J. Cagle, MD \\ Stryker: Consultor \\ Johnson \& Johnson: Consultor \\ Bradford O. Parsons, MD}

Arthrex: Consultor

Os seguintes profissionais não têm conflitos de interesse ou fontes de financiamento que precisem ser mencionados: Kevin C. Wang, Akshar V. Patel, Christopher A. White e Benjamin D. Gross

\section{Referências}

1 Anoushiravani AA, O'Connor CM, DiCaprio MR, Iorio R. Economic Impacts of the COVID-19 Crisis: An Orthopaedic Perspective. J Bone Joint Surg Am 2020;102(11):937-941

2 O'Connor CM, Anoushiravani AA, DiCaprio MR, Healy WL, Iorio R. Economic Recovery After the COVID-19 Pandemic: Resuming Elective Orthopedic Surgery and Total Joint Arthroplasty. J Arthroplasty 2020;35(7S):S32-S36

3 Mouton C, Hirschmann MT, Ollivier M, Seil R, Menetrey J. COVID19 - ESSKA guidelines and recommendations for resuming elective surgery. J Exp Orthop 2020;7(01):28

4 Vles GF, Ghijselings S, De Ryck I, et al. Returning to Elective Orthopedic Surgery During the COVID-19 Pandemic: A Multidis- ciplinary and Pragmatic Strategy for Initial Patient Selection. J Patient Saf 2020;16(04):e292-e298

5 Moverman MA, Puzzitiello RN, Pagani NR, Barnes CL, Jawa A, Menendez ME. Public Perceptions of Resuming Elective Surgery During the COVID-19 Pandemic. J Arthroplasty 2021;36(02): 397-402.e2

6 Simovitch RW, Friedman RJ, Cheung EV, et al. Rate of Improvement in Clinical Outcomes with Anatomic and Reverse Total Shoulder Arthroplasty. J Bone Joint Surg Am 2017;99(21): 1801-1811

7 Day JS, Lau E, Ong KL, Williams GR, Ramsey ML, Kurtz SM. Prevalence and projections of total shoulder and elbow arthroplasty in the United States to 2015. J Shoulder Elbow Surg 2010;19 (08):1115-1120

8 Cancienne JM, Brockmeier SF, Gulotta LV, Dines DM, Werner BC. Ambulatory Total Shoulder Arthroplasty: A Comprehensive Analysis of Current Trends, Complications, Readmissions, and Costs. J Bone Joint Surg Am 2017;99(08):629-637

9 Berman JE, Mata-Fink A, Kassam HF, Blaine TA, Kovacevic D. Predictors of Length of Stay and Discharge Disposition After Shoulder Arthroplasty: A Systematic Review. J Am Acad Orthop Surg 2019;27(15):e696-e701

10 Wong JSH, Cheung KMC. Impact of COVID-19 on Orthopaedic and Trauma Service: An Epidemiological Study. J Bone Joint Surg Am 2020;102(14):e80

11 Egol KA, Konda SR, Bird ML, et al; NYU COVID Hip Fracture Research Group. Increased Mortality and Major Complications in Hip Fracture Care During the COVID-19 Pandemic: A New York City Perspective. J Orthop Trauma 2020;34(08):395-402

12 Giorgi PD, Villa F, Gallazzi E, et al. The management of emergency spinal surgery during the COVID-19 pandemic in Italy. Bone Joint J 2020;102-B(06):671-676

13 Bixby EC, Boddapati V, Anderson MJJ, Mueller JD, Jobin CM, Levine WN. Trends in total shoulder arthroplasty from 2005 to 2018: lower complications rates and shorter lengths of stay despite patients with more comorbidities. JSES Int 2020;4(03): 657-661

14 Dunn JC, Lanzi J, Kusnezov N, Bader J, Waterman BR, Belmont PJ Jr. Predictors of length of stay after elective total shoulder arthroplasty in the United States. J Shoulder Elbow Surg 2015;24(05): 754-759

15 Edwards PK, Ebert JR, Littlewood C, Ackland T, Wang A. Effectiveness of formal physical therapy following total shoulder arthroplasty: A systematic review. Shoulder Elbow 2020;12(02): 136-143 